\title{
Effect of Monomer Structure on Ionic Conductivity in a Systematic Set of Polyester Electrolytes
}

\author{
Danielle M. Pesko, ${ }^{\text {a }}$ Yukyung Jung, ${ }^{b}$ Alexandra L. Hasan, ${ }^{a}$ Michael A. Webb,${ }^{c}$ Geoffrey W. \\ Coates, ${ }^{\mathrm{b}}$ Thomas F. Miller III, ${ }^{\mathrm{c}}$ and Nitash P. Balsara ${ }^{\mathrm{a}, \mathrm{d}, \mathrm{e}}$ \\ ${ }^{a}$ Department of Chemical and Biomolecular Engineering, University of California, Berkeley, \\ California 94720, USA \\ ${ }^{b}$ Department of Chemistry and Chemical Biology, Baker Laboratory, Cornell University, Ithaca, \\ New York 14853, USA \\ ${ }^{c}$ Division of Chemistry and Chemical Engineering, California Institute of Technology, \\ Pasadena, California 91125, USA \\ ${ }^{d}$ Materials Science Division, Lawrence Berkeley National Laboratory, Berkeley, California \\ 94720, USA \\ ${ }^{e}$ Environmental Energy Technology Division, Lawrence Berkeley National Laboratory, \\ Berkeley, California 94720, USA
}

\section{Keywords}

Ionic conductivity; polymer electrolyte; monomer structure; polyester; PEO 


\section{Abstract}

Polymer electrolytes may enable the next generation of lithium ion batteries with improved energy density and safety. Predicting the performance of new ion-conducting polymers is difficult because ion transport depends on a variety of interconnected factors which are affected by monomer structure: interactions between the polymer chains and the salt, extent of dissociation of the salt, and dynamics in the vicinity of ions. In an attempt to unravel these factors, we have conducted a systematic study of the dependence of monomer structure on ionic conductivity, $\sigma$, and glass transition temperature, $T_{\mathrm{g}}$, using electrolytes composed of aliphatic polyesters and lithium bis(trifluoromethanesulfonyl) imide (LiTFSI) salt. The properties of these electrolytes were compared to those of poly(ethylene oxide) (PEO), a standard polymer electrolyte for lithium batteries. We define a new measure of salt concentration, $\rho$, the number of lithium ions per unit length of the monomer backbone. This measure enables collapse of the dependence of both the $\sigma$ and $T_{\mathrm{g}}$ on salt concentration for all polymers (polyesters and PEO). Analysis based on the Vogel-Tammann-Fulcher (VTF) equation reveals the effect of different oxygen atoms on ion transport. The VTF fits were used to factor out the effect of segmental motion in order to clarify the relationship between molecular structure and ionic conductivity. While the conductivity of the newly-developed polyesters were lower than that of PEO, our study provides new insight into the relationship between ion transport and monomer structure in polymer electrolytes. 


\section{Introduction}

Replacing conventional organic liquid electrolytes with a nonflammable alternative is a crucial step toward safer rechargeable lithium batteries. Ion-conducting polymers are of particular interest in electrochemical applications due to their non-volatile nature and easily tunable properties.[1,2] Despite 40 years of persistent research, the ionic conductivity of the most promising solvent-free polymer electrolytes remain insufficient for use in commercial batteries. Our ability to design new and improved ion-conducting polymers is compromised by a lack of knowledge of the relationship between monomer structure and ion transport.

The mechanism that enables ion transport in polymers is inherently different from traditional liquids.[3-5] Solvent-free polymers solvate low lattice-energy salts through the formation of stable ion-polymer complexes;[6-8] hopping from one solvation complex to another is a mechanism that allows ions to travel through polymers.[9-11] Therefore, ionconducting polymers must contain polar groups which interact with at least one of the ions (typically the cation) to enable salt solvation,[12] but the strength of these interactions will impact hopping dynamics. The solvation of salt and the transport of ions are intrinsically coupled, and the type of polar groups present will directly affect the conductive properties of the material. Furthermore, the location and spacing of these groups influence the stiffness of the chains. More flexible chains exhibit rapid segmental motion which facilitates ion transport, and thus are desirable in polymer electrolytes.[13,14] Due to the complexity of these interrelated factors, determining relationship between molecular structure and ion transport remains an outstanding challenge.

A vast majority of the literature on polymer electrolytes is focused on mixtures of poly(ethylene oxide) (PEO) and lithium salts such as lithium bis(trifluoromethanesulfonyl) imide 
(LiTFSI) which exhibit reasonable ionic conductivities at temperatures above $60^{\circ} \mathrm{C}$, the melting temperature.[8,11,14-17] The ionic conductivity of PEO/LiTFSI in the vicinity of $80{ }^{\circ} \mathrm{C}$ is $1 \times 10^{-}$ ${ }^{3} \mathrm{~S} / \mathrm{cm},[15,16]$ significantly lower than the room temperature conductivity of liquid electrolytes used in current electric vehicles. The desire to obtain polymer electrolytes with improved lithium ion transport has motivated studies of conductivity in a variety of polymers such as polyesters,[18-22] polycarbonates,[23,24] polysiloxanes,[25-28] polyphosphazenes,[29] and perflouropolyethers.[30] Changes in the monomer structure affect the glass transition temperature, dielectric constant, ion solvation, salt dissociation, and ion hopping rates in ways that are, at this stage, difficult to predict. It is unclear which of these effects are responsible for the observed differences in conductivity due to the fact that the chemical structure of the monomers listed above are drastically different. Comparison between the results presented in references 18 -30 is further complicated by differences in salt used to create the electrolytes, salt concentration, and polymer molecular weight; the conductivity of polymer electrolytes is significantly affected by these parameters. To our knowledge, there is no framework that enables quantification of the factors the underlie ion transport in the chemically distinct polymers chains listed above.

In this paper, we have studied ion transport in a series of polyesters wherein the locations of the cation-solvating oxygen atoms in the monomer are systematically changed. The same salt was used in all the electrolytes and our study covers a wide range of overlapping salt concentrations. We ensured that the molecular weight of the polymers exceeded $4 \mathrm{~kg} / \mathrm{mol}$. In this regime the conductivity of PEO/LiTFSI mixtures is independent of chain length,[14,16] and the chemical identity of the endgroups.[31] We assume the same holds for the polyester electrolytes. 
We thus interpret differences in conductivity solely in terms of differences in the chemical structure of the monomers.

The chemical structure of the aliphatic polyesters used in this study are shown in Figure 1. We use two different backbones, one with an alkane link between the backbone ester groups, labeled $a$, and another with an ether link between the backbone ester groups, labeled $b$. Polymers labeled 1 have a methyl side chain, polymers labeled 2 have a methoxy-allyl side chain, and polymers labeled 3 have an ethylene-oxide sidechain with three ether oxygens. For completeness we also studied PEO. Careful consideration went into choosing these structures. We explore two polar groups: ethers and carbonyl-containing esters. Ethers are of particular interest in the polymer electrolyte community, while carbonyl groups are used in current lithium battery electrolytes.[5] In contrast to previous reports on polyesters,[18-22] the polymers in Figure 1 all possess sidechains of varying lengths. We chose to avoid linear polyesters to thwart crystallization; the comb polyesters in this study (Figure 1) are amorphous over the entire salt concentration and temperature ranges of interest.

\section{1a}

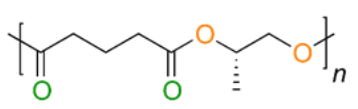

2a

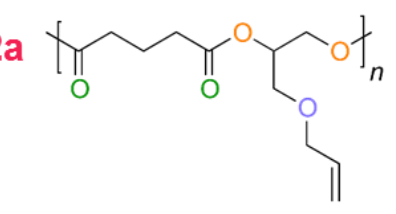

$3 a$

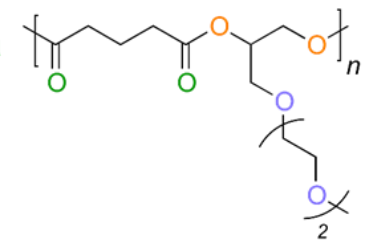

$1 b$

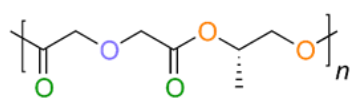

$2 b$

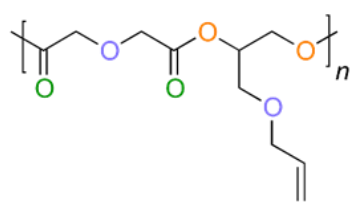

$3 b$

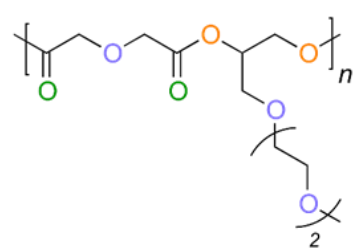

PEO

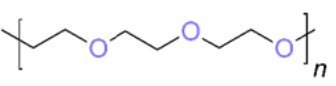


Figure 1. Structure and naming convention for polyesters and PEO. The monomer units for all polymers including PEO correspond to 9 atoms along the backbone. Oxygens are distinguished using color: carbonyl oxygens are green, ester oxygens are orange, and ether oxygens are purple.

The properties of the electrolytes listed in Figure 1 have been previously studied in the dilute salt concentration limit in reference 33. The focus of that work was to experimentally determine the dilute-ion transport characteristics of polyester electrolytes and utilize simulations for molecular-level insight describing the coordination environment and hopping mechanisms of a lithium ion. It is, perhaps, worth noting at the outset that the ionic conductivities of the newlydeveloped polyester electrolytes are less than that of PEO (at fixed temperature and salt concentration). The present study is mainly motivated by our desire to begin building a framework for understanding the relationship between monomer structure and ion transport. 


\section{Experimental Section}

2.1. Polymer Synthesis and Characterization. The polyesters used in this study were synthesized and characterized using methods described in reference 33 .

Table 1 provides the number-averaged molecular weight, $M_{\mathrm{n}}$, and polydispersity, $\oslash$, for each polymer.

2.2. Electrolyte Preparation. Electrolytes were prepared by mixing each polymer with lithium bis(trifluoromethanesulfonyl) imide (LiTFSI) salt. Due to the hygroscopic nature of LiTFSI, all sample preparation was carried out in an argon glovebox (MBraun) where $\mathrm{H}_{2} \mathrm{O}$ and $\mathrm{O}_{2}$ levels were maintained below $0.1 \mathrm{ppm}$ and $1 \mathrm{ppm}$ respectively. The set of six polyesters were dried, along with $5 \mathrm{~kg} / \mathrm{mol} \mathrm{PEO}$ (Polymer Source), at $90{ }^{\circ} \mathrm{C}$ under vacuum in the glovebox antechamber for a minimum of $8 \mathrm{~h}$, and then transferred into the glovebox. Dry polymer and LiTFSI salt (Novolyte) were dissolved into anhydrous N-methyl-2-pyrrolidone (NMP) and the solutions were mixed at $90{ }^{\circ} \mathrm{C}$ for a minimum of $5 \mathrm{~h}$. Once the solutes were fully dissolved, the caps were removed from the vials allowing NMP to evaporate and leave behind a homogeneous polymer/salt mixture. After drying on the hotplate at $90{ }^{\circ} \mathrm{C}$ for 2 days, the electrolytes were transferred to the glovebox antechamber and dried under vacuum for $8 \mathrm{~h}$ at $90{ }^{\circ} \mathrm{C}$ to remove any excess NMP. Most of the dry electrolytes were very viscous liquids at room temperature with the consistency of molasses. Electrolyte $1 \mathrm{~b}$ was solid-like at room temperature.

It is convenient to define concentration, $\rho$, as the molar ratio of lithium ions to polymer repeat units, $\rho=\left[\mathrm{Li}^{+} /\right.$monomer $]$, wherein the monomer is defined in Figure 1. It is perhaps worth noting that a PEO "monomer" is defined as having 3 repeating $\mathrm{CH}_{2}-\mathrm{CH}_{2}-\mathrm{O}$ units. The lengths of the backbones of all the monomers are thus comparable. Electrolytes with $\rho=0,0.02,0.04,0.08$, 
$0.12,0.16,0.2,0.24$, and 0.28 were created for each polyester and PEO. These values were chosen to span the full range of salt concentrations in an attempt identify the maximum conductivity of each polymer.

2.3. Differential Scanning Calorimetry. Samples were prepared by depositing 3-7 mg of each electrolyte into hermetically sealed aluminum pans. Differential scanning calorimetry (DSC) experiments were performed on a TA Instruments DSC Q200 instrument with the following temperature scan: heat to $110^{\circ} \mathrm{C}$ at $20^{\circ} \mathrm{C} / \mathrm{min}$, cool to $-75^{\circ} \mathrm{C}$ at $5^{\circ} \mathrm{C} / \mathrm{min}$, heat to $110{ }^{\circ} \mathrm{C}$ at 20 ${ }^{\circ} \mathrm{C} / \mathrm{min}$. The glass transition temperature, $T_{\mathrm{g}}$, values of the electrolytes were obtained from the second heating scan. $T_{\mathrm{g}}$ measurements were found to be repeatable within $1{ }^{\circ} \mathrm{C}$.

2.4. Electrochemical Measurements. Stainless steel symmetric cells were prepared for ionic conductivity measurements of electrolytes using ac impedance spectroscopy. Highly viscous liquid electrolytes were pressed into a $3.175 \mathrm{~mm}$ diameter hole within a $254 \mu \mathrm{m}$ thick silicone spacer. Two $200 \mu \mathrm{m}$ stainless steel electrodes were pressed on either side of the electrolyte-filled spacer. The silicone forms a good seal with stainless steel which prevents the polymers from leaking out of the cell. Due to a high $T_{\mathrm{g}}$, polymer $1 \mathrm{~b}$ was heated to $90{ }^{\circ} \mathrm{C}$ while pressing; all other electrolytes were soft enough to be pressed at room temperature. The thickness of each electrolyte was determined by measuring the thickness of the cell using a micrometer and subtracting the thickness of the electrodes. Aluminum tabs were secured to the electrodes using kapton tape. The entire cell was hermetically sealed within Showa-Denko pouch material leaving only the tab ends exposed. This allows for electrochemical measurements to take place outside of the glovebox while an air-free, water-free environment is maintained for the electrolyte.

Once removed from the glovebox, each cell was placed in a custom-built heating stage to determine conductivity in the range of $25{ }^{\circ} \mathrm{C}$ to $130{ }^{\circ} \mathrm{C}$. Complex impedance measurements were 
acquired using a Biologic VMP3 potentiostat for a frequency range of $1 \mathrm{~Hz}$ to $1 \mathrm{MHz}$ at an amplitude of $50 \mathrm{mV}$. Figure 2 shows a Nyquist plot of the impedance data obtained from polymer $1 \mathrm{~b}$ with $\rho=0.08$ at $90{ }^{\circ} \mathrm{C}$. As commonly observed with ion-conducting polymer electrolytes, there is a semicircle at high frequencies with a capacitive tail at low frequencies. The data was fit to an equivalent electrical circuit model that is suitable for finding resistance of a polymer electrolyte in a symmetric cell with blocking electrodes, shown in the inset of Figure 2. In this circuit, the parallel combination of $C_{\mathrm{b}}$, the capacitance of the bulk electrolyte, and $R_{\mathrm{b}}$, the bulk electrolyte resistance, effectively models the semicircle of the data; $Q_{\text {e, }}$ the pseudocapacitance (constant phase element) of the electrode accounts for the capacitive tail. Apparatus inductance, $L_{\mathrm{c}}$, and resistance, $R_{\mathrm{c}}$, were also included.

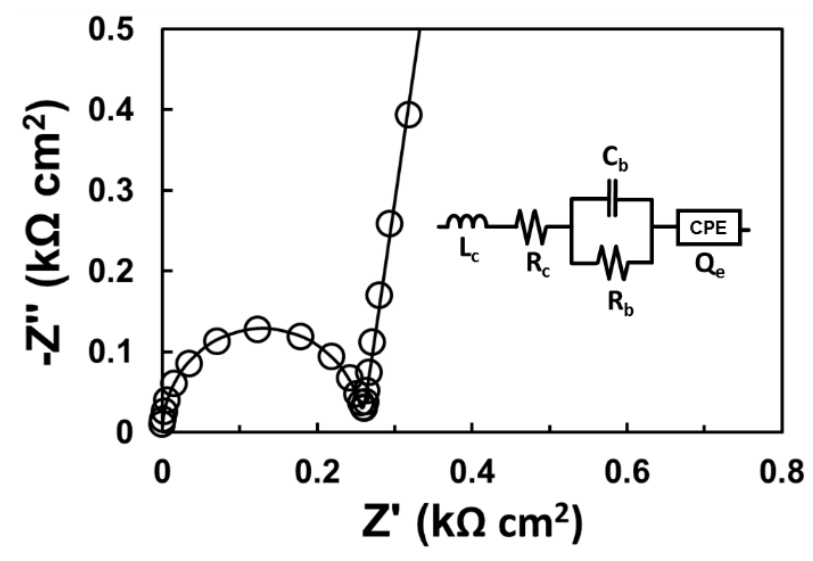

Figure 2. Nyquist impedance plot (-Z" vs $Z^{\prime}$ ) of polymer $1 \mathrm{~b}$ with $\rho=0.08$ at $90^{\circ} \mathrm{C}$ for a frequency range of $1 \mathrm{MHz}$ to $25 \mathrm{mHz}$. Open circles correspond to the experimental data and the solid line shows the least-squares fit obtained using the equivalent circuit shown in the inset.

Conductivity, $\sigma$, was then calculated using eq 1 , where $l$ is the electrolyte thickness, and $a$ is the electrolyte area.

$$
\sigma=\frac{l}{a R_{b}}
$$


Subsequent to conductivity measurements, each cell was disassembled in a glovebox and final thicknesses were measured. On average, the electrolyte thickness decreased $7 \%$ after annealing. The final sample thicknesses were used for the conductivity calculation. Finally, a visual inspection of the electrolyte was performed to ensure the samples had no bubbles or voids in the polymer. Such defects would alter the electrolyte volume and make conductivity calculations inaccurate, thus, these samples were discarded from the set. Error was calculated based on the standard deviation of three independent conductivity samples prepared for each electrolyte. 


\section{Results and Discussion}

Conductivity, $\sigma$, was measured at a wide range of salt concentrations and temperatures $\left(25-130^{\circ} \mathrm{C}\right)$ for each polymer. Results are shown in Figure 3 where conductivity of b-type polyesters and $\mathrm{PEO}$ obtained at $90{ }^{\circ} \mathrm{C}$ is plotted as a function of salt weight fraction, $w$. The atype polyesters have been excluded from this figure for clarity, but follow similar trends to their

b-type counterparts. For the polyesters, the highest conductivity occurs at $w$ values between 0.19 and 0.25 , whereas PEO exhibits a broad maximum at $w=0.34$. The reason for the non-monotonic dependence of conductivity on salt concentration is well established.[13,15] Conductivity increases with increasing salt concentration in the low concentration regime due to an increase in the number of charge carriers. However, screening effects become important with increasing salt concentration, and this reduces the number of "effective" charge carriers. In addition, interactions between polymer chains and salt molecules slow down segmental motion and this impedes ion transport. On the surface, the qualitative differences between the data obtained from polymers 1b, 2b, 3b, and PEO (Figure 3) seem to suggest that the interplay between charge carrier concentration and segmental motion in these systems is fundamentally different. 


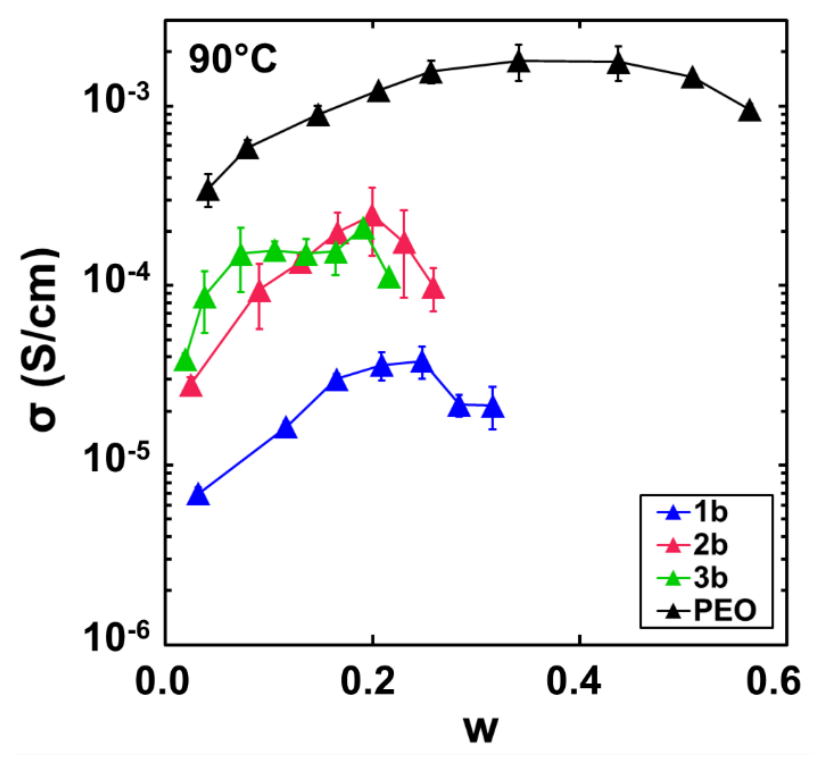

Figure 3. Conductivity, $\sigma$, as a function of weight fraction, $w$, of LiTFSI in each polymer. All data shown is at $90^{\circ} \mathrm{C}$.

The salt concentration in polymer electrolytes, particularly PEO, is often quantified by the ratio of lithium ions to ether oxygens, $r$.[16,32] In some cases, $1 / r$, the ratio of ether oxygens to lithium ions, is used to quantify salt concentration.[15,17] We prefer to use $r$ as it is directly related to molarity and molality which are standard measures of salt concentration in conventional electrolytes such as aqueous sulfuric acid. It is known, for example, that PEO/LiTFSI mixtures exhibit a maximum conductivity at $r=0.085 .[15,32]$ Such a ratio is suitable for describing salt concentration in polymers wherein the interactions between the salt molecules and all of the polar groups on the polymer chains are identical. In contrast to conventional polyethers, the polyesters investigated in this study have three different types of oxygens with varying electron densities (see Figure 1). Furthermore, the accessibility of the oxygens will depend on location; oxygens located on sidechains are expected to be more accessible than those located on the main chain. It is likely that the lack of collapse of the conductivity data shown in Figure 3 is due to these effects. Defining a measure of salt 
concentration that enables a collapse of the conductivity data has the potential to provide insight into the interactions between the different polar groups in the polyesters and LiTFSI.

Figure 4a shows the same data as Figure 3 with a new measure of salt concentration: $\rho$, the molar ratio of lithium ions to monomers defined in Figure 1. The abscissa of Figure 3 is restricted to $0.02 \leq \rho \leq 0.28$. It is evident that defining salt concentration in terms of $\rho$ aligns the conductivity trends for each polymer. In particular, the conductivity peaks occur at similar values of $\rho$ and the width of the peaks are also similar. In Figure $4 \mathrm{~b}$ we plot $\sigma / \sigma_{\max }$ versus $\rho$, where $\sigma_{\max }$ is the maximum conductivity of each polymer. The values of $\sigma_{\max }$ are given in

Table 1. We observe that all data sets collapse onto a master curve. Similar collapse is observed for conductivities measured between 40 and $130{ }^{\circ} \mathrm{C}$ (data not shown for brevity).

The maximum conductivity occurs at $\rho=0.22 \pm 0.02$ for all polyesters and PEO. This is noteworthy because the monomers as defined in Figure 1 contain varying numbers of oxygens, ranging from 3 to 8 . If the interactions between salt molecules and all of the oxygens were similar, then the conductivity maximum in polyesters $3 \mathrm{a}$ and $3 \mathrm{~b}$ would occur at $\rho$ values that were significantly higher than that of PEO. As seen in Figure 1, all monomers consist of 9 atoms along the backbone. Therefore our definition of $\rho$ can be thought of as the number of lithium ions per unit length of the monomer backbone. It appears that there is a spatial limitation in the heavily functionalized polyesters that prevents lithium ions from accessing all available oxygens. In contrast, PEO has only 3 oxygens per monomer, and all are readily accessible to solvate lithium.[33] We examined the possibility of using other definitions of salt concentration such as lithium ions per ether oxygen, lithium ions per carbonyl and ether oxygen, lithium ions per 
oxygen (including ester, carbonyl, and ether groups) and salt weight fraction. The conductivity data shown in Figure 4 do not collapse when these definitions of salt concentration are used.
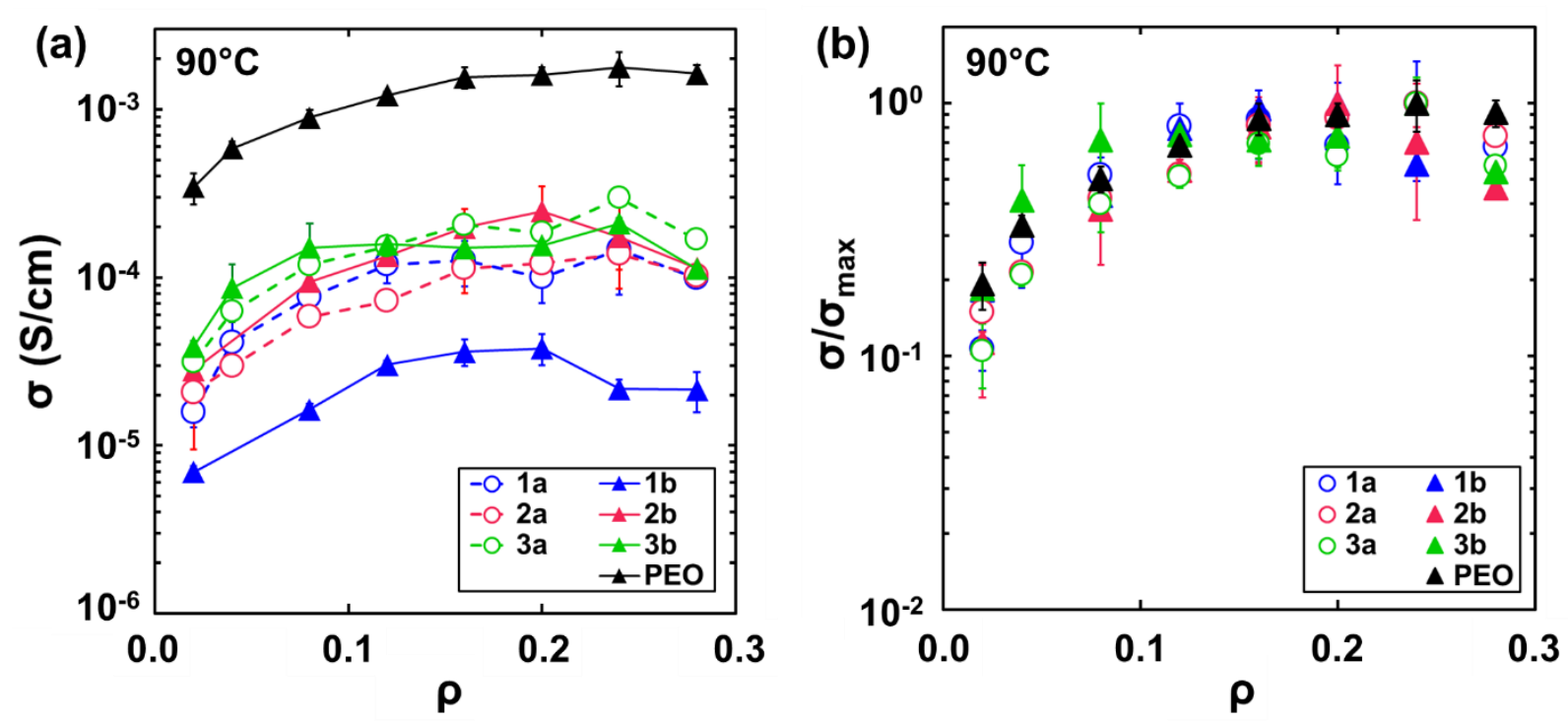

Figure 4. (a) Conductivity, $\sigma$, and (b) normalized conductivity, $\sigma / \sigma_{\max }$, as a function of $\rho$ at 90 ${ }^{\circ} \mathrm{C}$.

We find that $\sigma_{\max }$ for polymers $1 \mathrm{a}, 2 \mathrm{a}, 2 \mathrm{~b}, 3 \mathrm{a}$, and $3 \mathrm{~b}$ all fall in the range of $1.4 \times 10^{-4}$ to $3.0 \times 10^{-4} \mathrm{~S} / \mathrm{cm}$. The outlier of the polyester set is polymer $1 \mathrm{~b}$, which has a $\sigma_{\max }$ approximately one order of magnitude lower than all other polyesters. The glass transition temperature of our polymers at $\rho=0, \rho=0.02$, and $\rho=0.2$ are listed in

Table 1. We find that the $T_{\mathrm{g}}$ of polymer $1 \mathrm{~b}$ is significantly higher than that of the other polymers in all cases, thus the low value of $\sigma_{\max }$ of polymer $1 \mathrm{~b}$ is related to slow segmental motion. The most conductive polymer, PEO, has the lowest $T_{\mathrm{g}}$. There is, however, no correlation between $T_{\mathrm{g}}$ and $\sigma_{\max }$ for polymers with intermediate conductivity. It is evident that factors other than segmental motion are important for ion transport. 
Table 1: Material properties of polymers and VTF parameters of electrolytes at $\rho=0.02$ and $\rho=0.2$.

\begin{tabular}{ccccccccccc}
\hline Polymer & $\begin{array}{c}M_{\mathrm{n}} \\
(\mathrm{kg} / \mathrm{mol})\end{array}$ & $\begin{array}{c}\sigma_{\mathrm{max}, 90^{\circ} \mathrm{C}} \\
(\mathrm{S} / \mathrm{cm})\end{array}$ & $\begin{array}{c}T_{\mathrm{g}, \text { neat }} \\
\left({ }^{\circ} \mathrm{C}\right)\end{array}$ & $\begin{array}{c}T_{\mathrm{g}, \rho=0.02} \\
\left({ }^{\circ} \mathrm{C}\right)\end{array}$ & $\begin{array}{c}T_{\mathrm{g}, \mathrm{r}=0.2} \\
\left({ }^{\circ} \mathrm{C}\right)\end{array}$ & $\begin{array}{c}E_{\mathrm{a}, \rho=0.02} \\
(\mathrm{~kJ} / \mathrm{mol})\end{array}$ & $\begin{array}{c}A_{\rho=0.02} \\
\left(\mathrm{SK}^{1 / 2} / \mathrm{cm}\right)\end{array}$ & $\begin{array}{c}E_{\mathrm{a}, \rho=0.2} \\
(\mathrm{~kJ} / \mathrm{mol})\end{array}$ & $\begin{array}{c}A_{\rho=0.2} \\
\left(\mathrm{SK}^{1 / 2} / \mathrm{cm}\right)\end{array}$ \\
\hline 1a & 8.8 & 1.9 & $1.5 \times 10^{-4}$ & -28 & -25 & -12 & $8.6 \pm 1.1$ & $0.1 \pm 0.2$ & $9.2 \pm 1.2$ & $2.6 \pm 0.2$ \\
2a & 10.4 & 2.0 & $1.4 \times 10^{-4}$ & -41 & -40 & -26 & $8.3 \pm 1.5$ & $0.1 \pm 0.5$ & $8.5 \pm 1.0$ & $1.1 \pm 0.1$ \\
3a & 4.2 & 1.3 & $3.0 \times 10^{-4}$ & -44 & -43 & -28 & $8.6 \pm 1.3$ & $0.2 \pm 0.3$ & $9.2 \pm 1.1$ & $2.5 \pm 0.1$ \\
1b & 6.7 & 1.6 & $3.8 \times 10^{-5}$ & 12 & 14 & 26 & $10.7 \pm 1.2$ & $3.5 \pm 0.2$ & $8.9 \pm 1.4$ & $7.7 \pm 0.2$ \\
2b & 8.9 & 1.5 & $2.5 \times 10^{-4}$ & -14 & -13 & 0 & $8.7 \pm 1.1$ & $0.5 \pm 0.1$ & $9.6 \pm 2.3$ & $16.6 \pm 0.8$ \\
3b & 6.1 & 1.8 & $2.1 \times 10^{-4}$ & -25 & -23 & -12 & $9.2 \pm 1.1$ & $0.6 \pm 0.1$ & $9.8 \pm 1.2$ & $6.4 \pm 0.2$ \\
PEO & 5.0 & 1.1 & $1.8 \times 10^{-3}$ & -60 & -59 & -44 & $7.1 \pm 1.6$ & $0.5 \pm 0.8$ & $8.4 \pm 1.2$ & $7.2 \pm 0.2$ \\
\hline
\end{tabular}

The glass transition temperature of polymer electrolytes usually increases with salt concentration.[15,17] In many studies, the glass transition of mixtures is correlated with the weight fractions of the components.[34,35] In Figure 5a we plot $\Delta T_{\mathrm{g}}$, the increase in $T_{\mathrm{g}}$ at a given salt concentration relative to the neat sample, versus $w$. Included in this plot are data obtained from all six polyesters in the range $0 \leq \rho \leq 0.28$. The data obtained from PEO samples are limited due to experimental difficulties. Similar difficulties have been noted in previous studies of the glass transition in PEO.[15,36] In particular, we were not able to detect a $T_{\mathrm{g}}$ for the neat sample of PEO due to a high degree of crystallinity. We performed DSC on a $4.6 \mathrm{~kg} / \mathrm{mol}$ PEO (Sigma Aldrich) with a high polydispersity and obtained $T_{\mathrm{g}}=-60{ }^{\circ} \mathrm{C}$. We do not expect segmental motion of the polydisperse sample to be significantly different from that of the low polydispersity PEO used in our conductivity experiments. Lacking a better alternative, we assume that $T_{\mathrm{g}}$ of pure PEO is $-60{ }^{\circ} \mathrm{C}$. For similar reasons we were unable to determine $T_{\mathrm{g}}$ of PEO/LiTFSI mixtures from $\rho=0.02$ to $\rho=0.16$. At values of $\rho>0.16$, PEO/LiTFSI mixtures are amorphous and determining $T_{\mathrm{g}}$ was straightforward. It is evident that $\Delta T_{\mathrm{g}}$ at a given value of $w$ 
depends on the type of polymer, particularly at high concentrations (Figure 5a). In Figure 5b we plot $\Delta T_{\mathrm{g}}$ versus $\rho$. The data from all samples collapse onto a straight line, $\Delta T_{\mathrm{g}}=67.7 \rho$, which we obtained using a least-squares fit through the data.

Determining a definition of salt concentration that leads to a collapse in both $\sigma$ and $\Delta T_{\mathrm{g}}$ is the first step in relating monomer structure and ion transport. We show below that analysis of data obtained at a fixed value of $\rho$ provides insight regarding the polar groups responsible for salt complexation and steric effects that limit access to certain polar groups.
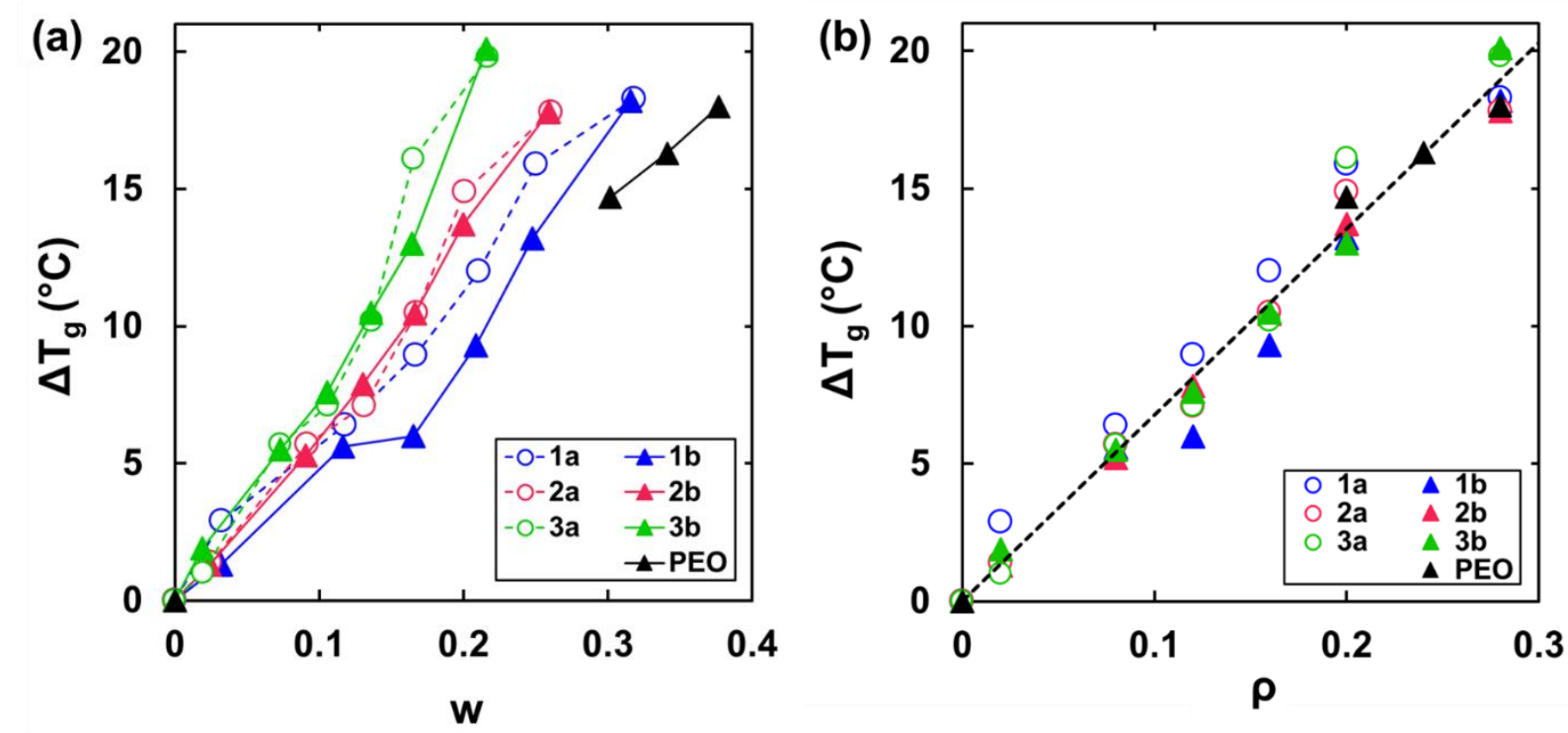

Figure 5. Increase in $T_{\mathrm{g}}$ as a function of (a) weight fraction, $w$, and (b) $\rho$ for the polyester and PEO electrolytes. The $T_{\mathrm{g}}$ at $w=0$ and $\rho=0$ is that of the neat polymer.

The ionic conductivity of polymer electrolytes is influenced by a number of interrelated factors: interactions between monomers on the polymer chain and the salt species, extent of dissociation of the salt, and chain conformations and dynamics, i.e. segmental motion, in the vicinity of ions.[13,15] We use the well-known Vogel-Tammann-Fulcher (VTF) equation to factor out the effect of segmental motion on conductivity.[37] The temperature dependence of conductivity of polymer electrolytes is given by the VTF equation, 


$$
\sigma=A T^{-1 / 2} \exp \left(\frac{-E_{\mathrm{a}}}{R\left(T-T_{\mathrm{o}}\right)}\right)
$$

In this equation $T_{\mathrm{o}}$ is a reference temperature, $R$ is the universal gas constant, and $A$ and $E_{\mathrm{a}}$ are VTF parameters obtained by fitting experimental data. The reference temperature, $T_{\mathrm{o}}$, is taken to be $50{ }^{\circ} \mathrm{C}$ below the glass transition temperature of the electrolyte. The parameter $A$ is often related to the concentration of charge carriers in the system, and $E_{\mathrm{a}}$ is the effective activation energy for ion transport.

In Figure 6a we plot $\sigma$ versus $1000 /\left(T-T_{\mathrm{g}}+50\right)$ for $\rho=0.02$, a dilute salt concentration. The data from all of our samples are approximately linear when plotted in this format indicating agreement with the VTF equation. Note that $T_{\mathrm{g}}$ is the glass transition of the electrolyte with $\rho=0.02$

Table 1). The parameters $A$ and $E_{\mathrm{a}}$, obtained by least-squares fits through the data in Figure 6a, are given in

Table 1. Despite large differences in the chemical formulae of the monomers, $E_{\mathrm{a}}$ values are similar, ranging from 7.1 to $10.7 \mathrm{~kJ} / \mathrm{mol}($

Table 1). In other words, the VTF plots of all of the polymers are nearly parallel (Figure 6a). The vertical offset of the VTF plots of different polymers mainly reflects the magnitude of A. The VTF data of the set of polymers examined here in the dilute limit are segregated into three groups: (1) PEO, (2) b-type polymers, and (3) a-type polymers, in order of decreasing 
conductivity at fixed $T-T_{\mathrm{g}}$. The main difference between a-type and b-type polymers is the addition of an ether oxygen on the backbone of b-type polymers (Figure 1). We thus conclude that this backbone ether oxygen plays an important role in ion transport through the b-type polymers.

In Figure $6 \mathrm{~b}$ we plot $\sigma$ versus $1000 /\left(T-T_{\mathrm{g}}+50\right)$ for $\rho=0.2$, the concentration in the vicinity of the maximum conductivity. The values of $T_{\mathrm{g}}, A$, and $E_{\mathrm{a}}$ for $\rho=0.2$ are provided in

Table 1. We find that the data of all samples are approximately linear and parallel, and the values of $E_{\mathrm{a}}$ are comparable to those obtained from the data in Figure 6a. We thus conclude that the activated process of ion hopping does not significantly change as a function of either polymer type or salt concentration. The VTF data of the set of polymers examined here in the concentrated limit are segregated into two groups: (1) PEO, 1b, and 2b, and (2) 3b and the a-type polymers, in order of decreasing conductivity at fixed $T-T_{\mathrm{g}}$. Polymer $3 \mathrm{~b}$ appears to be an outlier of the polyesters, aligning with b-type polymers in the dilute limit and with a-type polymers in the concentrated limit.

Comparison between Figure $6 \mathrm{a}$ and Figure $6 \mathrm{~b}$ indicate that the trends observed in the VTF plots change as a function of salt concentration. The VTF plot of PEO at $\rho=0.02$ lies above that obtained from the polyesters. In contrast, the VTF plot of PEO at $\rho=0.2$ was coincident with $1 \mathrm{~b}$ and $2 \mathrm{~b}$. In addition, the VTF plot of $3 \mathrm{~b}$ at $\rho=0.02$ is coincident with that of the other b-type polyesters; in contrast, the VTF plot of $3 \mathrm{~b}$ at $\rho=0.2$ lies below that of $1 \mathrm{~b}$ and $2 \mathrm{~b}$. These differences suggest that the factors that underpin ion transport in the dilute and concentrated regimes may be different in some cases (e.g. PEO and 3b). 

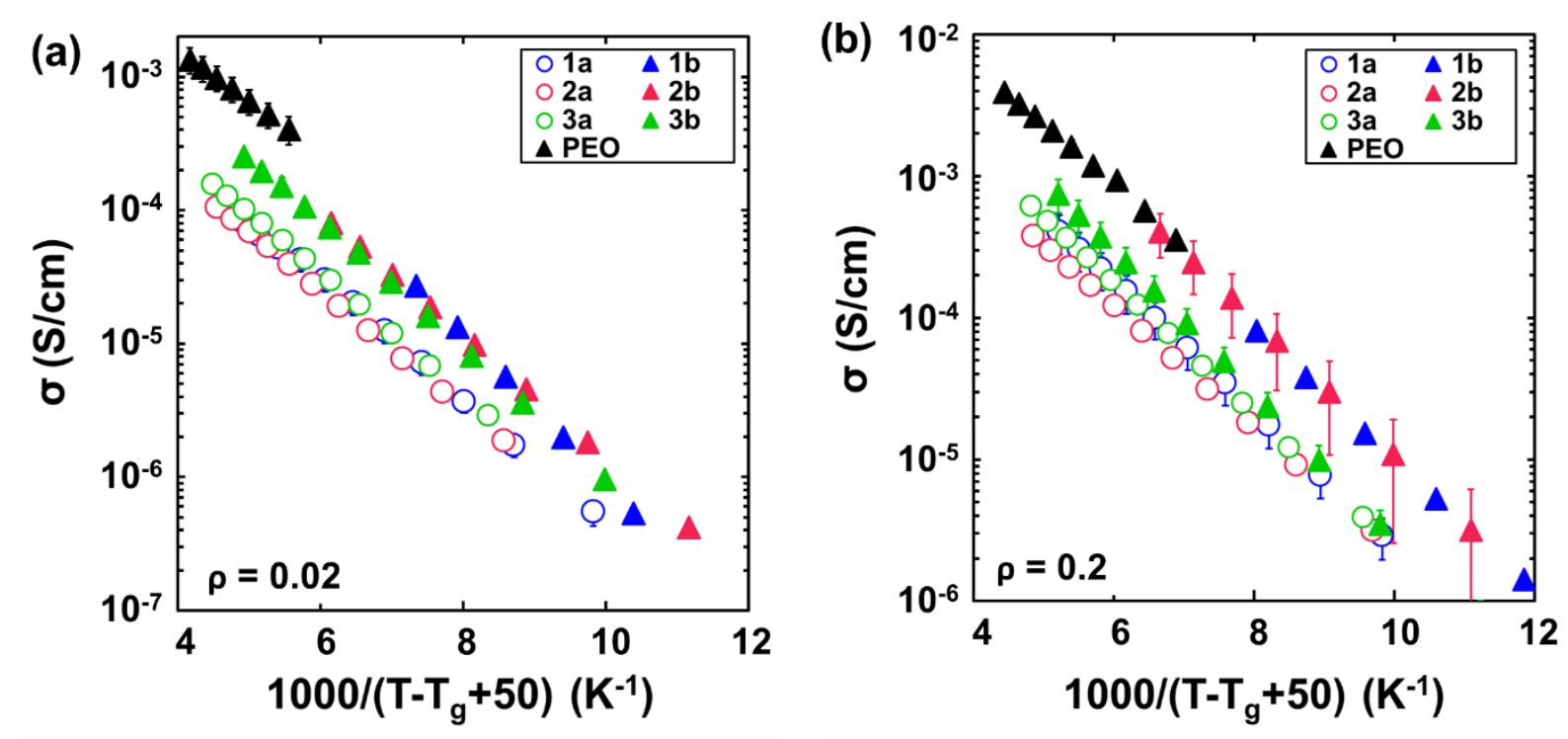

Figure 6. Vogel-Tammann-Fulcher plot of conductivity of electrolytes at (a) $\rho=0.02$ and (b) $\rho=0.2$. In both figures, the data for PEO is limited to temperatures above the melting point.

To further investigate the effect of salt concentration on ion transport, we use the VTF fit parameters, $E_{\mathrm{a}}$ and $A$ (Table 1 and 2 in SI), obtained from the temperature dependent data to calculate a reduced conductivity, $\sigma_{\mathrm{r}}$, at a set temperature $(75 \mathrm{~K})$ away from $T_{\mathrm{g}}$.

$$
\sigma_{\mathrm{r}}=A\left(T_{\mathrm{g}}+75 \mathrm{~K}\right)^{-1 / 2} \exp \left(\frac{-E_{\mathrm{a}}}{R(125 \mathrm{~K})}\right)
$$

Here, $T_{\mathrm{g}}$ is the measured glass transition temperature of the polymer/salt mixture of interest. In the range $0<\rho<0.16$, we assume that $T_{\mathrm{g}}$ of PEO is given by the linear fit in Figure $5 \mathrm{~b}$. Since the VTF lines for the polymers are essentially parallel, the dependence of $\sigma_{\mathrm{r}}$ on $\rho$ is qualitatively similar at all values of $T-T_{\mathrm{g}}$. In Figure 7 we show the dependence of $\sigma_{\mathrm{r}}$ on $\rho$ for all the polymers. At first glance, Figure 7 appears to be similar to Figure 4a. There are, however, important differences that shed light on the underlying factors that affect ion transport. The maxima obtained in $\sigma$ versus $\rho$ plots of all seven polymers (Figure 4a) are not present in the $\sigma_{\mathrm{r}}$ versus $\rho$ plot (Figure 7). The only outlier is polymer $2 \mathrm{~b}$ wherein a maximum is observed in the $\sigma_{\mathrm{r}}$ versus $\rho$ 
plot. It is clear that the maxima in $\sigma$ versus $\rho$ plots were obtained due to increasing $T_{\mathrm{g}}$ with increasing salt concentration. When this effect is factored out, most $\sigma_{\mathrm{r}}$ versus $\rho$ plots appear to reach plateaus above $\rho=0.2$ (except for 2b). At low $\rho$, all polymers show an increase in conductivity due to increasing charge carrier concentration. Above $\rho=0.2$ the solvation sites become saturated and the reduced conductivity becomes independent of salt concentration. Another significant difference between Figure 4a and 7 is the vertical offset between different polymers. The distinction between a-type and b-type polymers in $\sigma$ versus $\rho$ plots is unclear (Figure 4a). In contrast, all of the b-type polymers lie above a-type polymers in $\sigma_{\mathrm{r}}$ versus $\rho$ plots (Figure 7). It is evident that factoring out the effect of the glass transition temperature clarifies the relationship between molecular structure and ion transport. At fixed distance from $T_{\mathrm{g}}$, all btype polymers have higher conductivity than the a-type polymers at all values of $\rho$. Figure 7 shows that at low $\rho, 3 \mathrm{~b}$ is as conductive as $1 \mathrm{~b}$ and $2 \mathrm{~b}$, but above $\rho=0.08$, the conductivity of $3 \mathrm{~b}$ falls close to the a-type polymers. At low $\rho$, PEO is more conductive than the b-type polyesters above $\rho<0.16$; these differences diminish with increasing salt concentration.

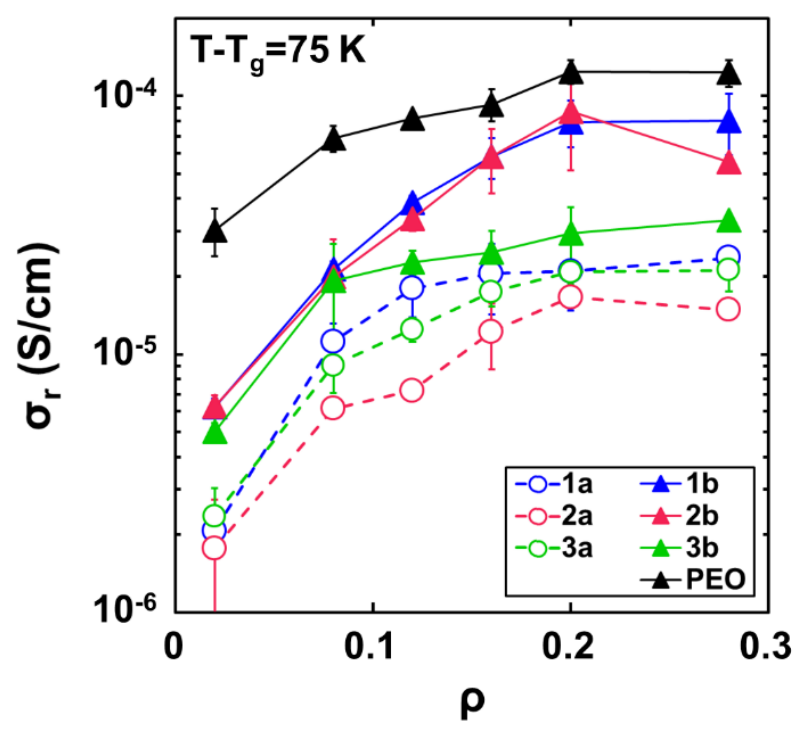


Figure 7. Reduced conductivity at $T-T_{g}=75 \mathrm{~K}$, or $1000 /\left(T-T_{g}+50\right)=8 \mathrm{~K}^{-1}$, as a function of $\rho$. Error bars have been adapted from the conductivity error (Figure $4 \mathrm{a}$ ). 


\section{Conclusions}

Ion transport in polymer electrolytes depends on a variety of interconnected factors: interactions between the polymer matrix and the salt species (dissociated and undissociated), extent of dissociation of the salt, and chain conformations and dynamics, i.e. segmental motion, in the vicinity of ions. Systematic changes in the monomer structure enable unraveling of these effects. Here, we study ion transport in a set of aliphatic polyester electrolytes and present an approach for analyzing conductivity data that provides insight regarding the relationship between monomer structure and ion transport.

We define a new variable for salt concentration, $\rho$, which enables collapse of both the $\sigma$ and $T_{\mathrm{g}}$ measurements for all polymers. This allows us to extract information about the polymersalt interactions and determine that some oxygens in the heavily functionalized polyesters are inaccessible. A Vogel-Tammann-Fulcher (VTF) analysis reveals the effect of different oxygens on ion transport: backbone ether oxygens enhance conductivity in the absence of sidechain ether oxygens. The VTF fits are used to calculate the reduced conductivity of our electrolytes, $\sigma_{\mathrm{r}}$, wherein the effect of segmental motion is factored out. The a-type and b-type polymers are clearly segregated when compared on $\sigma_{\mathrm{r}}$ versus $\rho$ plots, and PEO lies above the polyesters at all values of $\rho$. When segmental motion is factored out, the differences in reduced conductivity reflect differences in the dissociation of the salt, solvation environment of the cation, and ion hopping. Distinguishing between these factors will require input from other techniques such as neutron diffraction, NMR, and X-ray absorption spectroscopy. We hope to use these techniques in future studies to further our understanding of the relationship between monomer structure and ion transport in polymer electrolytes. 


\section{Acknowledgements}

This research was supported by the National Science Foundation under DMREF Award Number NSF-CHE-1335486. DSC experiments were performed at the Molecular Foundry user facilities at Lawrence Berkeley National Laboratory supported by the Office of Science, Office of Basic

Energy Sciences, of the U.S. Department of Energy under Contract No. DE-AC02-05CH11231.

\section{Author Information}

Corresponding Author

*E-mail: nbalsara@berkeley.edu.

\section{Notes}

The authors declare no competing financial interest. 


\section{Abbreviations}

PEO poly(ethylene oxide)

LiTFSI lithium bis(trifluoromethanesulfonyl) imide

NMP N-methyl-2-pyrrolidone

DSC differential scanning calorimetry

VTF Vogel-Tammann-Fulcher

$C_{\mathrm{b}} \quad$ capacitance of the bulk electrolyte (F)

$R_{\mathrm{b}} \quad$ bulk electrolyte resistance $(\Omega)$

$Q_{\mathrm{e}} \quad$ pseudo-capacitance of the electrodes (F)

$L_{\mathrm{c}} \quad$ apparatus inductance $(\mathrm{H})$

$R_{\mathrm{c}} \quad$ apparatus resistance $(\Omega)$

$l \quad$ electrolyte thickness $(\mathrm{cm})$

a electrolyte area $\left(\mathrm{cm}^{2}\right)$

$\sigma \quad$ conductivity $(\mathrm{S} / \mathrm{cm})$

$\sigma_{\max } \quad \operatorname{maximum}$ conductivity $(\mathrm{S} / \mathrm{cm})$

w salt weight fraction

$r \quad$ molar ratio of $\mathrm{Li}^{+}$to ether oxygens

$\rho \quad$ molar ratio of $\mathrm{Li}^{+}$to monomer

??n number-averaged molecular weight $(\mathrm{kg} / \mathrm{mol})$

$Ð$ polydispersity

$T_{\mathrm{g}} \quad$ glass transition temperature $\left({ }^{\circ} \mathrm{C}\right)$

$\Delta T_{\mathrm{g}} \quad$ increase in $T_{\mathrm{g}}$ compared to neat polymer $\left({ }^{\circ} \mathrm{C}\right)$

$T$ temperature $\left({ }^{\circ} \mathrm{C}\right)$

$T_{\mathrm{o}} \quad$ reference temperature $\left({ }^{\circ} \mathrm{C}\right)$

$R \quad$ universal gas constant $(\mathrm{kJ} / \mathrm{mol} \mathrm{K})$

$A \quad$ VTF prefactor $\left(\mathrm{SK}^{1 / 2} / \mathrm{cm}\right)$

$E_{\mathrm{a}} \quad$ effective activation energy $(\mathrm{kJ} / \mathrm{mol})$

$\sigma_{\mathrm{r}} \quad$ reduced conductivity $(\mathrm{S} / \mathrm{cm})$ 


\section{References}

[1] B. Scrosati, J. Garche, J. Power Sources 195 (2010) 2419-2430.

[2] B. Scrosati, C.A. Vincent, MRS Bull. 25 (2011) 28-30.

[3] D. Baril, Solid State Ionics 94 (1997) 35-47.

[4] J.W. Fergus, J. Power Sources 195 (2010) 4554-4569.

[5] K. Xu, Chem. Rev. 104 (2004) 4303-4417.

[6] D.E. Fenton, J.M. Parker, P.V. Wright, Polymer (Guildf). 14 (1973) 589.

[7] D. Shriver, B. Papke, M. Ratner, R. Dupon, T. Wong, M. Brodwin, Solid State Ionics 5 (1981) 8388.

[8] O. Borodin, G.D. Smith, Macromolecules 31 (1998) 8396-8406.

[9] M.A. Ratner, D.F. Shriver, Chem. Rev. 88 (1988) 109-124.

[10] S.D. Druger, A. Nitzan, M.A. Ratner, J. Chem. Phys. 79 (1983) 3133-3142.

[11] O. Borodin, G.D. Smith, Macromolecules 39 (2006) 1620-1629.

[12] C.A. Vincent, Prog. Solid State Chem. 17 (1987) 145-261.

[13] A. Killis, J. LeNest, H. Cheradame, A. Gandini, Die Makromol. Chemie 183 (1982) 2835-2845.

[14] J. Shi, C. Vincent, Solid State Ionics 60 (1993) 11-17.

[15] S. Lascaud, M. Perrier, A. Vallee, S. Besner, J. Prud'homme, M. Armand, Macromolecules 27 (1994) 7469-7477.

[16] A.A. Teran, M.H. Tang, S.A. Mullin, N.P. Balsara, Solid State Ionics 203 (2011) 18-21.

[17] W. Gorecki, M. Jeannin, E. Belorizky, C. Roux, M. Armand, J. Phys. Condens. Matter 7 (1995) 6823-6832.

[18] R.D. Armstrong, M.D. Clarke, Electrochim. Acta 29 (1984) 1443-1446.

[19] R. Dupon, J. Electrochem. Soc. 131 (1984) 586-589.

[20] M. Watanabe, M. Rikukawa, K. Sanui, N. Ogata, H. Kato, T. Kobayashi, Z. Ohtaki, Macromolecules 17 (1984) 2902-2908.

[21] M. Watanabe, M. Togo, K. Sanui, N. Ogata, T. Kobayashi, Z. Ohtaki, Macromolecules 17 (1984) 2908-2912.

[22] Y. Lee, Solid State Ionics 138 (2001) 273-276.

[23] Y. Tominaga, T. Shimomura, M. Nakamura, Polymer (Guildf). 51 (2010) 4295-4298.

[24] M. Smith, Solid State Ionics 140 (2001) 345-351.

[25] K. Nagaoka, H. Naruse, I. Shinohara, M. Watanabe, J. Polym. Sci. Polym. Lett. Ed. 22 (1984) 659-663.

[26] R. Hooper, L.J. Lyons, D.A. Moline, R. West, Organometallics 18 (1999) 3249-3251.

[27] R. Hooper, L.J. Lyons, M.K. Mapes, D. Schumacher, D.A. Moline, R. West, Macromolecules 34 (2001) 931-936.

[28] C.P. Fonseca, S. Neves, J. Power Sources 104 (2002) 85-89.

[29] P. Blonsky, D. Shriver, P. Austin, H. Allcock, Solid State Ionics 18-19 (1986) 258-264.

[30] D.H.C. Wong, J.L. Thelen, Y. Fu, D. Devaux, A.A. Pandya, V.S. Battaglia, N.P. Balsara, J.M. 
DeSimone, Proc. Natl. Acad. Sci. U. S. A. 111 (2014) 3327-31.

[31] D. Devaux, R. Bouchet, D. Glé, R. Denoyel, Solid State Ionics 227 (2012) 119-127.

[32] M. Singh, O. Odusanya, G.M. Wilmes, H.B. Eitouni, E.D. Gomez, A.J. Patel, V.L. Chen, M.J. Park, P. Fragouli, H. Iatrou, N. Hadjichristidis, D. Cookson, N.P. Balsara, Macromolecules 40 (2007) 4578-4585.

[33] M.A. Webb, Y. Jung, D.M. Pesko, B.M. Savoie, U. Yamamoto, G.W. Coates, N.P. Balsara, Z.-G. Wang, T.F. Miller, ACS Cent. Sci. 1 (2015) 198-205.

[34] T.G. Fox, Bull. Am. Phys. Soc. 1 (1956) 22060-26218.

[35] P.R. Couchman, Macromolecules 11 (1978) 1156-1161.

[36] J.A. Faucher, J. Appl. Phys. 37 (1966) 3962-3964.

[37] M.B. Armand, J.M. Chabagno, M.J. Duclot, in:, Fast Ion Transp. Solids. Electrodes Electrolytes, 1979, pp. 131-136. 